\title{
Inference of termination conditions for numerical loops in Prolog
}

\author{
Alexander Serebrenik ${ }^{\star}$,Danny De Schreye \\ Department of Computer Science, K.U. Leuven \\ Celestijnenlaan 200A, B-3001, Heverlee, Belgium \\ E-mail: \{Alexander.Serebrenik, Danny.DeSchreye\}@cs.kuleuven.ac.be
}

\section{Introduction}

Numerical computations form an essential part of almost any real-world program. Clearly, in order for a termination analyser to be of practical use it should contain a mechanism for inferring termination of such computations. However, this topic attracted less attention of the research community. In this work we concentrate on automatic termination inference for logic programs depending on numerical computations. Dershowitz et al. [8] showed that termination of general numerical computations, for instance on floating point numbers, may be counter-intuitive, i.e., the observed behaviour does not necessarily coincide with the theoretically expected one. Thus, we restrict ourselves to integer computations only.

While discussing termination of integer computations the following question should be asked: what conditions on the queries should be assumed, such that the queries will terminate. We refer to this question as the termination inference problem.

Example 1. $p(X) \leftarrow X<7, X 1$ is $X+1, p(X 1)$. This program terminates for queries $p(X)$, for all integer values of $X$. Thus, the answer for the termination inference problem is the condition "true".

This example also hints at why the traditional approaches to termination analysis fail to prove termination of this example. These approaches are mostly based on the notion of level mapping, that is, a function from the set of all possible atoms to the natural numbers, which should decrease while traversing the rules. In our case, such a level mapping should depend on $X$, but $X$ can be negative as well!

Two approaches for solving this problem are possible. First, one can change the definition of the level mapping to map atoms to integers. However, integers are, in general, not well-founded. To prove termination one

\footnotetext{
* supported by GOA: " $L P^{+}$: a second generation logic programming language".
} 
should prove that the mapping is to some well-founded subset of integers. In the example above $(-\infty, 7)$ forms such a subset with an ordering $\succ$, such that $x \succ y$ if $x<y$, with respect to the usual ordering on integers.

The second approach that we present in the paper does not require changing the definition of level mapping. Indeed, the level mapping as required exists. It maps $p(X)$ to $7-X$ if $X<7$ and to 0 otherwise. This level mapping decreases while traversing the rule, i.e., the size of $p(X)$, $7-X$, is greater than the size of $p(X 1), 6-X$, thus, proving termination. We present a transformation that allows us to define such a level mappings in an automatic way by incorporating techniques of [8], such as level mapping inference, in the well-known framework of the acceptability with respect to a set $[5,6]$. This integration provides not only a better understanding of termination behaviour of integer computations, but also the possibility to perform the analysis automatically as in Decorte et al. [7].

The rest of the paper is organised as follows. After making some preliminary remarks, we present in Section 3 our transformation-first by means of an example, then more formally. In Section 4 we discuss more practical issues and present the algorithm implementing the termination inference. In Section 5 we discuss further extensions, such as proving termination of programs depending in numerical computations as well as symbolic ones. Then we review related work and conclude.

\section{Preliminaries}

We follow the standard notation for terms and atoms. A query is a finite sequence of atoms. Given an atom $A, \operatorname{rel}(A)$ denotes the predicate occuring in $A$. Atom $P$ denotes a set of all atoms that can be constructed from the language underlying $P$. The extended base $B_{P}^{E}$ is a quotient set of Atom $_{P}$ modulo the variant relation. An SLD-tree constructed using the left-to-right selection rule of Prolog is called an LD-tree. A goal $G$ $L D$-terminates for a program $P$, if the $\mathrm{LD}$-tree for $(P, G)$ is finite.

Definition 1. [1] Let $P$ be a program and $p, q$ be predicates occuring in it. We say that $p$ refers to $q$ in $P$ if there is a clause in $P$ that uses $p$ in its head and $q$ in its body; that $p$ depends on $q$ in $P$ and write $p \sqsupseteq q$, if $(p, q)$ is in the transitive, reflexive closure of the relation refers to; and that $p$ and $q$ are mutually recursive and write $p \simeq q$, if $p \sqsupseteq q$ and $q \sqsupseteq p$.

We recall some basic notions, related to termination analysis. A level mapping is a function $|\cdot|: B_{P}^{E} \rightarrow \mathcal{N}$, where $\mathcal{N}$ is the set of the naturals.

We study termination of programs with respect to sets of queries. The following notion is one of the most basic notions in this framework. 
Definition 2. [6] Let $P$ be a definite program and $S$ be a set of atomic queries. The call set, $C a l l(P, S)$, is the set of all atoms $A$, such that a variant of $A$ is a selected atom in some derivation for $P \cup\{\leftarrow Q\}$, for some $Q \in S$ and under the left-to-right selection rule.

The following definition [14] generalises the notion of acceptability with respect to a set $[5,6]$ by extending it to mutual recursion.

Definition 3. Let $S$ be a set of atomic queries and $P$ a definite program. $P$ is acceptable with respect to $S$ if there exists a level mapping $|\cdot|$ such that for any $A \in \operatorname{Call}(P, S)$, for any $A^{\prime} \leftarrow B_{1}, \ldots, B_{n}$ in $P$, such that $\operatorname{mgu}\left(A, A^{\prime}\right)=\theta$ exists, for any $B_{i}$, such that $\operatorname{rel}\left(B_{i}\right) \simeq \operatorname{rel}(A)$ and for any c.a.s. $\sigma$ for $\leftarrow\left(B_{1}, \ldots, B_{i-1}\right) \theta$ holds that $|A|>\left|B_{i} \theta \sigma\right|$.

De Schreye et al. [5] characterise LD-termination in terms of acceptability.

Theorem 1. (cf. [5]) Let $P$ be a program. $P$ is acceptable with respect to a set $S$ if and only if $P$ is LD-terminating for all queries in $S$.

We also need to introduce the notion of interargument relations.

Definition 4. [7] Let $P$ be a definite program, $p / n$ a predicate in $P$. An interargument relation for $p / n$ is $R_{p} \subseteq \mathcal{N}^{n}$. $R_{p}$ is a valid interargument relation for $p / n$ with respect to a norm $\|\cdot\|$ if and only if for every $p\left(t_{1}, \ldots, t_{n}\right) \in$ Atom $_{P}$ if $P \models p\left(t_{1}, \ldots, t_{n}\right)$ then $\left(\left\|t_{1}\right\|, \ldots,\left\|t_{n}\right\|\right) \in R_{p}$.

To characterise program transformations Bossi and Cocco [3] introduced the following notion for a program $P$ and a query $Q: \mathcal{M} \llbracket P \rrbracket(Q)=$ $\{\sigma \mid$ there is a successful LD-derivation of $Q$ and $P$ with c.a.s. $\sigma\} \cup\{\perp \mid$ there is an infinite LD-derivation of $Q$ and $P\}$.

\section{Methodology}

In this section we introduce our methodology using a simple example. In the subsequent sections, we formalise it and discuss different extensions.

The following example generates an oscillating sequence and stops if the generated value is greater than 1000 or smaller than -1000 .

Example 2. We are interested in proving termination of the set of queries $S=\{p(z) \mid z$ is an integer $\}$ with respect to the following program:

$p(X) \leftarrow X>1, X<1000, X 1$ is $-X * X, p(X 1)$.

$p(X) \leftarrow X<-1, X>-1000, X 1$ is $X * X, p(X 1)$.

The direct attempt to define the level mapping of $p(X)$ as $X$ fails, since $X$ can be positive as well as negative. Thus, a more complex level mapping should be defined. We start with some observations. 
The first clause is applicable if $1<X<1000$, the second one, if $-1000<X<-1$. Thus, termination of $p(X)$ for $X \leq-1000,-1 \leq X \leq$ 1 or $X \geq 1000$ is trivial. Moreover, if an infinite sequence is obtained by applying the first clause at the first step, then for the recursive call $p(X 1)$, it holds that $-1000<X 1<-1$ and if the second clause was applied at the first step of the infinite sequence, then for the recursive call $p(X 1)$, it holds that $1<X 1<1000$. We use this observation and replace a predicate $p$ with two new predicates $p^{1<X<1000}$ and $p^{-1000<X<-1}$, such that $p^{1<X<1000}$ is called if $p(X)$ is called and $1<X<1000$ holds and $p^{-1000<X<-1}$ is called if $p(X)$ is called and $-1000<X<-1$ holds. The following program is obtained:

$p^{1<X<1000}(X) \leftarrow X>1, X<1000, X 1$ is $-X * X, p^{-1000<X<-1}(X 1)$. $p^{-1000<X<-1}(X) \leftarrow X<-1, X>-1000, X 1$ is $X * X, p^{1<X<1000}(X 1)$.

Now we define two different level mappings, one for atoms of $p^{1<X<1000}$ and another one for atoms of $p^{-1000<X<-1}$. Let $\left|p^{1<X<1000}(n)\right|=1000-$ $n$ if $1<n<1000$ and 0 otherwise and let $\left|p^{-1000<X<-1}(n)\right|=1000+$ $n$ if $-1000<n<-1$ and 0 otherwise. We verify acceptability of the transformed program with respect to $\left\{p^{1<X<1000}(n) \mid 1<n<1000\right\} \cup$ $\left\{p^{-1000<X<-1}(n) \mid-1000<n<-1\right\}$. This implies termination of the transformed program with respect to these queries, and thus, termination of the original program with respect to $S$.

Due to the lack of space we discuss only queries of the form $p^{1<X<1000}(n)$ for $1<n<1000$. The only clause that its head can be unified with this query is the first clause. The only atom of a predicate mutually recursive with $p^{1<X<1000}$ is $p^{-1000<X<-1}(m)$. Then, $\left|p^{1<X<1000}(n)\right|>$ $\left|p^{-1000<X<-1}(m)\right|$ should hold ${ }^{1}$, i.e., $1000-n>1000+m$, that is $1000-n>1000-n^{2}\left(n>1\right.$ and $\left.m=-n^{2}\right)$, which is true for $n>1$.

The intuitive presentation above hints at the major issues to be discussed in the following sections: how the cases such as those above can be extracted from the program, and how the program should be transformed.

\subsection{Basic notions}

In this section we formally introduce some notions that further analysis will be based on. Recall that the aim of our analysis is to find, given a predicate and a query, a sufficient condition for termination of this query with respect to this program. Thus, we need to define a notion of a termination condition. We start with a number of auxiliary definitions.

\footnotetext{
${ }^{1}$ The clause is applicable only if $1<n<1000$. Thus, $\left|p^{-1000<X<-1}(n)\right|=1000+n$.
} 
Definition 5. Let $p$ be a predicate of arity $n$. Then, $\$ 1^{p}, \ldots, \$ n^{p}$ are called argument position denominators.

If the predicate is clear from the context the superscripts will be omitted.

Definition 6. Let $P$ be a program, $S$ be a set of queries. An argument position $i$ of a predicate $p$ is called integer argument position, if for every $p\left(t_{1}, \ldots, t_{n}\right) \in \operatorname{Call}(P, S), t_{i}$ is an integer.

Argument position denominators corresponding to integer argument positions will be called integer argument position denominators.

An integer inequality is an atom of one of the following forms $\operatorname{Exp} 1>$ $\operatorname{Exp} 2, \operatorname{Exp} 1<\operatorname{Exp} 2, \operatorname{Exp} 1 \geq \operatorname{Exp} 2$ or $\operatorname{Exp} 1 \leq \operatorname{Exp} 2$, where $\operatorname{Exp} 1$ and Exp2 are constructed from integers, variables and the four operations of arithmetics. A symbolic inequality over the arguments of a predicate $p$ is constructed similarly to an integer inequality. However, instead of variables, integer argument positions denominators are used.

Example 3. $X>0$ and $Y \leq X+5$ are integer inequalities. Given a predicate $p$ of arity 3 , having only integer argument positions $\$ 1^{p}>0$ and $\$ 2^{p} \leq \$ 1^{p}+\$ 3^{p}$ are symbolic inequalities over the arguments of $p$.

Disjunctions of conjunctions based on integer inequalities are called integer conditions. Similarly, propositional calculus formulae based on symbolic inequalities over the arguments of the same predicate are called symbolic conditions over the integer arguments of this predicate.

Definition 7. Let $p\left(t_{1}, \ldots, t_{n}\right)$ be an atom and let $c_{p}$ be a symbolic condition over the arguments of $p$. An instance of the condition with respect to an atom, $c_{p}\left(p\left(t_{1}, \ldots, t_{n}\right)\right)$, is obtained by replacing the argument positions denominators with the corresponding arguments, i.e., $\$ i^{p}$ with $t_{i}$.

Example 4. Let $p(X, Y, 5)$ be an atom and let $c_{p}$ be $\left(\$ 1^{p}>0\right) \wedge\left(\$ 2^{p} \leq\right.$ $\left.\$ 1^{p}+\$ 3^{p}\right)$. Then, $c_{p}(p(X, Y, 5))$ is $(X>0) \wedge(Y \leq X+5)$.

Now we are ready to define termination condition formally.

Definition 8. Let $P$ be a program, and $Q$ be an atomic query. A symbolic condition $c_{\text {rel }(Q)}$ is a termination condition for $Q$ if given that $c_{\operatorname{rel}(Q)}(Q)$ holds, $Q$ left-terminates with respect to $P$.

A termination condition for Example 2 is true, i.e., $p(X)$ terminates for every integer $X$. We'll see further that this is not always the case. 
We discuss now inferring what values integer arguments can take during traversal of the rules, i.e., the "case analysis" performed in Example 2. It provides already the underlying intuition-calls of the predicate $p^{c}$ are identical to the calls of the predicate $p$, where $c$ holds for its arguments. More formally, we define a notion of set of adornments. Later we specify when it is guard-tuned and we show how such a guard-tuned set of adornments can be constructed.

Definition 9. Let $p$ be a predicate. The set $\mathcal{A}_{p}=\left\{c_{1}, \ldots, c_{n}\right\}$ of symbolic conditions over the integer arguments of $p$ is called set of adornments for $p$ if for all $i, j$ such that $1 \leq i<j \leq n, c_{i} \wedge c_{j}=$ false and $\bigvee_{i=1}^{n} c_{i}=$ true.

Example 5. Example 2, continued. The following are examples of sets of adornments: $\{\$ 1 \leq 100, \$ 1>100\}$ and $\{(\$ 1 \leq-1000) \vee(-1 \leq \$ 1 \leq$ 1) $\vee(\$ 1 \geq 1000),-1000<\$ 1<-1,1<\$ 1<1000\}$.

\subsection{Program transformation}

The next question that should be answered is how the program should be transformed given a set of adornments. After this transformation $p^{c}\left(X_{1}, \ldots, X_{n}\right)$ will behave with respect to the transformed program exactly as $p\left(X_{1}, \ldots, X_{n}\right)$ does, for all calls that satisfy the condition $c$. To define a transformation formally we introduce the following definition:

Let $H \leftarrow B_{1}, \ldots, B_{n}$ be a rule. $B_{1}, \ldots, B_{i}$, is called prefix of the rule, if for all $j, 1 \leq j \leq i \leq n, B_{j}$ is an integer inequality and the only variables in its arguments are variables of $H . B_{1}, \ldots, B_{i}$ is called the maximal prefix of the rule, if it is a prefix and $B_{1}, \ldots, B_{i}, B_{i+1}$ is not a prefix.

Since a prefix constrains only variables appearing in the head of a clause there exists a symbolic condition over the arguments of the predicate of the head, such that the prefix is its instance with respect to the head. In general, this symbolic condition is not necessarily unique. The following notion guarantees uniqueness of such symbolic conditions. In this case we say that the symbolic condition corresponds to the prefix.

Definition 10. [8] A rule $H \leftarrow B_{1}, \ldots, B_{n}$ is called partially normalised if all integer argument positions in $H$ are occupied by distinct variables ${ }^{2}$.

We will also say that a program $P$ is partially normalised if all the rules in $P$ are partially normalised. After integer argument positions are identified a program can be easily rewritten to partially normalised form.

Now we are ready to present the transformation formally.

\footnotetext{
${ }^{2}$ If such a rule has only integer arguments Apt et al. [2] call it homogeneous.
} 
Definition 11. Let $P$ be a program and let $p$ be a predicate in it. Let $\mathcal{A}=\bigcup_{q \in P} \mathcal{A}_{q}$ be a set of possible adornments for $P$. Then, the program $P^{a}$, called adorned with respect to $p$, is obtained in two steps as following:

1. For every rule $r$ in $P$, for every subgoal $q\left(t_{1}, \ldots, t_{n}\right)$ in the body of $r$, s.t. $p \simeq q$ and for every $A \in \mathcal{A}_{q}$ replace $q\left(t_{1}, \ldots, t_{n}\right)$ by $q^{A}\left(t_{1}, \ldots, t_{n}\right)$.

2. For every rule $r$

Are adornments and inequalities in the body of $r$ consistent? * If not-reject the rule.

If $r$ defines some $q$, such that $q \simeq p$

Get as adornments of the head of $r$ all $A \in \mathcal{A}_{q}$, that are consistent with comparisons of the maximal prefix of $r$ and adornments of the body of $r$.

Example 6. Example 2, continued. The sets of adornments presented in Example 5 are used. With the first set of adornments we obtain $P^{a_{1}}$ :

$p^{\$ 1 \leq 100}(X) \leftarrow X>1, X<1000, X 1$ is $-X * X, p^{\$ 1 \leq 100}(X 1)$.

$p^{\$ 1>100}(X) \leftarrow X>1, X<1000, X 1$ is $-X * X, p^{\$ 1 \leq 100}(X 1)$.

$p^{\$ 1 \leq 100}(X) \leftarrow X<-1, X>-1000, X 1$ is $X * X, p^{\$ 1 \leq 100}(X 1)$.

$p^{\$ 1 \leq 100}(X) \leftarrow X<-1, X>-1000, X 1$ is $X * X, p^{\$ 1>100}(X 1)$.

If the second set of adornments is used, the program $P^{a_{2}}$ is obtained:

$$
\begin{aligned}
& p^{1<\$ 1<1000}(X) \leftarrow X>1, X<1000, X 1 \text { is }-X * X, \\
& p^{-1000<\$ 1<-1}(X 1) \text {. } \\
& p^{1<\$ 1<1000}(X) \leftarrow X>1, X<1000, X 1 \text { is }-X * X, \\
& p^{(\$ 1 \leq-1000) \vee(-1 \leq \$ 1 \leq 1) \vee(\$ 1 \geq 1000)}(X 1) . \\
& p^{-1000<\$ 1<-1}(X) \leftarrow X<-1, X>-1000, X 1 \text { is } X * X \text {, } \\
& p^{1<\$ 1<1000}(X 1) . \\
& p^{-1000<\$ 1<-1}(X) \leftarrow X<-1, X>-1000, X 1 \text { is } X * X \text {, } \\
& p^{(\$ 1 \leq-1000) \vee(-1 \leq \$ 1 \leq 1) \vee(\$ 1 \geq 1000)}(X 1) .
\end{aligned}
$$

Correctness of the transformation should be proved. Finiteness of the number of clauses, the number of subgoals in a clause and the number of elements in an adornment ensure that the transformation terminates. Next we need to prove that the transformation preserves termination.

Adorning clauses introduces new predicates. This means that the query $Q$ gives rise to a number of different queries. Clearly, termination of all of these queries with respect to $P^{a}$ is equivalent to termination of $Q$ with respect to $P^{a}$ augmented by a set of the clauses, such that for every $p \simeq \operatorname{rel}(Q)$ and for every $A \in \mathcal{A}_{p}$ the clause $p\left(X_{1}, \ldots, X_{n}\right) \leftarrow$ $p^{A}\left(X_{1}, \ldots, X_{n}\right)$ is added. We call this extended program $P^{a g}$. 
Lemma 1. Let $P$ be a program, and let $Q$ be a query. Let $P^{a g}$ be a program obtained as described above. Then, $\mathcal{M} \llbracket P^{a g} \rrbracket(Q) \subseteq \mathcal{M} \llbracket P \rrbracket(Q)$.

Proof. For all proofs we refer to [13].

The second direction of the containment depends on the consistency check strategy applied at the point marked by $*$ in the definition of $P^{a}$.

Example 7. Let $Q$ be $p(X)$ and let $P$ be the following program:

$p(X) \leftarrow X>0, q(X), X<0 . \quad q(X) \leftarrow X>0, p(X)$.

Predicates $p$ and $q$ are mutually recursive. Thus, both of them should be adorned. Let $\mathcal{A}_{p}$ be $\{\$ 1>0, \$ 1 \leq 0\}$ and $\mathcal{A}_{q}$ be $\{\$ 1>0, \$ 1 \leq 0\}$. The following program is obtained after the first step of the adorning process. $p(X) \leftarrow X>0, q^{\$ 1>0}(X), X<0 . \quad q(X) \leftarrow X>0, p^{\$ 1>0}(X)$. $p(X) \leftarrow X>0, q^{\$ 1 \leq 0}(X), X<0 . \quad q(X) \leftarrow X>0, p^{\$ 1 \leq 0}(X)$.

The second step of the adorning process infers adornments for the heads of the clauses, possibly rejecting the inconsistent ones. If the inference technique tries to use all the information it has in the body constraints and adornments of body subgoals, a program $\left\{q^{\$ 1>0}(X) \leftarrow X>0, p^{\$ 1>0}(X)\right\}$ is obtained. Other clauses are rejected because of the inconsistency. The query $p(X)$ terminates with respect to the extended program while it does not terminate with respect to the original one. Thus, such an inference technique can actually improve termination.

In order for termination to be preserved a weaker inference engine should be used, for example, considering inequalities only of the maximal prefix. Then the following is obtained: $p^{\$ 1>0}(X) \leftarrow X>0, q^{\$ 1>0}(X), X<$ 0. $q^{\$ 1>0}(X) \leftarrow X>0, p^{\$ 1>0}(X)$. The query $p(X)$ does not terminate with respect to the extended program as expected. The following lemma shows that if this weaker inference is used, termination is preserved.

Lemma 2. Let $P$ be a program, and let $Q$ be a query. Let $P^{a g}$ be a program obtained as described above. Then, $\mathcal{M} \llbracket P \rrbracket(Q) \subseteq \mathcal{M} \llbracket P^{a g} \rrbracket(Q)$.

For the case of maximal prefixes the following summarises the results.

Theorem 2. Let $P$ be a program, let $Q$ be a query and let $\mathcal{A}$ be a set of adornments. Let $P^{a g}$ be a program obtained as described above with respect to $\mathcal{A}$. Then, $\mathcal{M} \llbracket P \rrbracket(Q)=\mathcal{M} \llbracket P^{a g} \rrbracket(Q)$.

This theorem has two important corollaries.

Corollary 1. Let $P$ be a program, let $Q$ be a query and let $\mathcal{A}$ be a set of adornments. Let $A=\left\{a \mid a \in \mathcal{A}\right.$, for all $q$ such that $\operatorname{rel}(Q)^{a} \sqsupseteq q$ : $q$ is not recursive in $\left.P^{a}\right\}$. Then $\bigvee_{a \in A}$ is a termination condition for $P$. 
Example 8. Example 6, continued. In $P^{a_{2}} p^{(\$ 1 \leq-1000) \vee(-1 \leq \$ 1 \leq 1) \vee(\$ 1 \geq 1000)}$ does not depend on recursive predicates. By the corollary, $(\$ 1 \leq-1000) \vee$ $(-1 \leq \$ 1 \leq 1) \vee(\$ 1 \geq 1000)$ is a termination condition for $p(X)$.

Theorem 2 implies that a program $P$ is LD-terminating with respect to all queries in a set of atomic queries $S$ if and only if $P^{a g}$, constructed as above, is acceptable with respect to $S$. The latter is equivalent to acceptability of $P^{a}$ with respect to $\left\{q^{A}\left(t_{1}, \ldots, t_{n}\right) \mid q\left(t_{1}, \ldots, t_{n}\right) \in S, A \in \mathcal{A}_{q}\right\}$.

Corollary 2. Let $P$ be a program, $S$ be a set of atomic queries and $\mathcal{A}=$ $\bigcup_{Q \in S, q \simeq \operatorname{rel}(Q)} \mathcal{A}_{q}$ be a set of adornments. Let $P^{a}$ be obtained with respect to $\mathcal{A}$. $P$ is LD-terminating with respect to all queries in $S$ if and only if $P^{a}$ is acceptable with respect to $\left\{q^{A}\left(t_{1}, \ldots, t_{n}\right) \mid q\left(t_{1}, \ldots, t_{n}\right) \in S, A \in \mathcal{A}_{q}\right\}$.

This corollary allows us to complete the termination proof for Example 2.

Example 9. We show that $P^{a_{2}}$ is acceptable with respect to the set $S=$ $\left\{p^{(\$ 1 \leq-1000) \vee(-1 \leq \$ 1 \leq 1) \vee(\$ 1 \geq 1000)}(X), p^{1<\$ 1<1000}(X), p^{-1000<\$ 1<-1}(X)\right\}$.

Then, $S=\operatorname{Call}\left(P^{a_{2}}, S\right)$. Let $|\cdot|$ be defined as: $\left|p^{-1000<\$ 1<-1}(X)\right|=$ $1000+X$, if $-1000<X<-1$ and 0 otherwise; $\left|p^{1<\$ 1<1000}(X)\right|=1000-$ $X$ if $1<X<1000$ and 0 otherwise; $\left|p^{(\$ 1 \leq-1000) \vee(-1 \leq \$ 1 \leq 1) \vee(\$ 1 \geq 1000)}(X)\right|$ $=0$.

We do not prove completely that $P^{a_{2}}$ is acceptable with respect to $S$ via $|\cdot|$, but analyse only one call, $p^{1<\$ 1<1000}(X)$. There are two clauses, (1) and (2), such that their heads can be unified with it. (2) is not recursive and the condition holds vacuously. (1) is recursive and acceptability requires $\left|p^{1<\$ 1<1000}(X)\right|>\left|p^{-1000<\$ 1<-1}\left(-X^{2}\right)\right|$, where $1<X<1000$. If $-X^{2} \leq-1000$ then $\left|p^{-1000<\$ 1<-1}\left(-X^{2}\right)\right|=0$ and descent is clear. Otherwise, $\left|p^{-1000<\$ 1<-1}\left(-X^{2}\right)\right|=1000-X^{2}$, where $0<1000-X^{2}<1000-X$, since $X>1$. As before, there is descent in the level mapping. The other calls are proved similarly.

\section{Practical issues}

In the previous section we have shown the transformation that allows reasoning on termination of the numerical computations. In this section we discuss a number of practical issues to be considered for automation.

\subsection{Guard-tuned sets of adornments}

In Example 5 we have seen two different sets of adornments. Both of them are valid according to Definition 9. However, $\{-1000<\$ 1<-1,1<$ 
$\$ 1<1000,(\$ 1 \leq-1000) \vee(-1 \leq \$ 1 \leq 1) \vee(\$ 1 \geq 1000)\}$ is in some sense preferable to $\{\$ 1 \leq 100, \$ 1>100\}$. First of all, it has a declarative reading: the sets that are constructed express conditions that, when satisfied, allow traversing the rule. Second, observe that $P^{a_{1}}$ has not only two mutually recursive predicates, as $P^{a_{2}}$ does, but also self-loop on one of the predicates. To distinguish between "better" and "worse" sets of adornments we define guard-tuned sets of adornments.

Definition 12. Let $P$ be a partially normalised program, let $p$ be a predicate in $P$. A set of adornments $\mathcal{A}_{p}$ is called guard-tuned if for every $A \in \mathcal{A}_{p}$ and for every rule $r \in P$ with the symbolic condition c corresponding to its maximal prefix, either $c \wedge A=$ false or $c \wedge A=A$ holds.

Example 10. The first set of adornments, presented in Example 5, is not guard-tuned while the second one is guard-tuned.

Examples 5 and 10 suggest two ways of constructing a guard-tuned set of adornments. Given a program $P$ one might collect the symbolic conditions, corresponding to the maximal prefixes of the rules defining a predicate $p$ (we denote this set $\mathcal{C}_{p}$ ) and add the completion of the constructed disjunction. Unfortunately, this set is not necessarily a set of adornments and if so, it is not necessary guard-tuned.

Example 11. $r(X) \leftarrow X>$ 5. $r(X) \leftarrow X>10, r(X)$. Two sets of symbolic conditions can be constructed: $\left\{r^{\$ 1 \leq 5}, r^{\$ 1>5}, r^{\$ 1>10}\right\}$ which is not a set of adornments and $\left\{r^{\$ 1 \leq 5}, r^{\$ 1>5}\right\}$ which is not guard-tuned.

We use a different approach. First, we find $\mathcal{C}_{p}=\left\{c_{1}, \ldots, c_{n}\right\}$. Then we define $\mathcal{A}_{p}$ to be the set of conjunctions of $c_{i}$ 's and their negations. We claim that the constructed set is always a guard-tuned set of adornments.

Example 12. As above, $\mathcal{C}_{p}=\{\$ 1>5, \$ 1>10\}$. After simplifying and removing inconsistencies $\mathcal{A}_{p}=\{\$ 1>10, \$ 1>5 \wedge \$ 1 \leq 10, \$ 1 \leq 5\}$.

Lemma 3. Let $P$ be a program, $p$ be a predicate in $P$ and $\mathcal{A}_{p}$ be constructed as described. Then $\mathcal{A}_{p}$ is a guard-tuned set of adornments.

\subsection{How to define a level mapping?}

The problem with defining level mappings is that they should reflect changes on possibly negative arguments and remain non-negative at the same time. We also like to remain in the framework of level mappings on atoms defined as linear combinations of sizes of their arguments. 
Definition 13. Let $p^{E_{1} \rho E_{2}}$ be an adorned predicate, where $E_{1}$ and $E_{2}$ are expressions and $\rho \in\{>, \geq\}$. The primitive level mapping is defined as: $\quad\left|p^{E_{1} \rho E_{2}}\left(t_{1}, \ldots, t_{n}\right)\right|^{p r}=\left(E_{1}-E_{2}\right)\left(t_{1}, \ldots, t_{n}\right)$

if $E_{1}\left(t_{1}, \ldots, t_{n}\right) \rho E_{2}\left(t_{1}, \ldots, t_{n}\right)$ and 0 otherwise.

If more than one conjunct appears in the adornment, the level mapping is defined as a linear combination of primitive level mappings corresponding to the conjuncts. If a conjunct is a disjunction, it is ignored, since disjunctions are introduced only if some rule cannot be applied.

Definition 14. Let $p^{c}$ be an adorned predicate, The natural level mapping is: $\left|p^{c}\left(t_{1}, \ldots, t_{n}\right)\right|=\sum_{E_{1} \rho E_{2} \in c} c_{E_{1} \rho E_{2}}\left|p^{E_{1} \rho E_{2}}\left(t_{1}, \ldots, t_{n}\right)\right|^{p r}$, where the c's are natural number coefficients, $E_{1}, E_{2}$ and $\rho$ are as above.

Example 13. The level mappings used in Example 9 are natural level mappings such that $c_{\$ 1>1}=c_{\$ 1<-1}=0, c_{\$ 1<1000}=c_{\$ 1>-1000}=1$. For $p^{(\$ 1 \leq-1000) \vee(-1 \leq \$ 1 \leq 1) \vee(\$ 1 \geq 1000)}$ the definition holds trivially.

The approach of [7] defines symbolic counterparts of the level mappings and infers the values of the coefficients by solving a system of constraints.

\subsection{Inferring termination constraints}

In this section, we combine the steps studied so far to an algorithm that infers termination conditions. The termination condition is constructed as a disjunction of two: $c_{1}$ for non-recursive cases, according to Corollary 1 , and $c_{2}$, for recursive cases, incrementally refined by adding to conjunction constraints on the integer variables, obtained from the acceptability condition, as in $[7]^{3}$. The algorithm is presented in Figure 1.

Example 14. $q(X, Y) \leftarrow X>Y, Z$ is $X-Y, q(Z, Y)$.

We look for values of $X$ and $Y$ such that $q(X, Y)$ terminates. First, the algorithm infers adornments. In our case $\{\$ 1>\$ 2, \$ 1 \leq \$ 2\}$ are inferred.

The adorned version of this program is $q^{\$ 1>\$ 2}(X, Y) \leftarrow X>Y, Z$ is $X-Y, q^{\$ 1>\$ 2}(Z, Y)$.

$q^{\$ 1>\$ 2}(X, Y) \leftarrow X>Y, Z$ is $X-Y, q^{\$ 1 \leq \$ 2}(Z, Y)$.

There is no clause defining $q^{\$ 1 \leq \$ 2}$. By Corollary $1, \$ 1 \leq \$ 2$ is a termination condition. This is the one we denoted $c_{1}$. The termination condition for $q^{\$ 1>\$ 2}$, denoted $c_{2}$ is initialised to be $\$ 1>\$ 2$. The level mapping is $\left|q^{\$ 1>\$ 2}(X, Y)\right|=c_{\$ 1>\$ 2}(X-Y)$ if $X>Y$ and 0 otherwise. The acceptability decrease implies (see [7]): $c_{\$ 1>\$ 2}(X-Y)>c_{\$ 1>\$ 2}((X-Y)-Y)$,

\footnotetext{
${ }^{3}$ Any other technique proving termination and able provide some constraint that, if satisfied, implies termination can be used instead of [7].
} 
Let $P$ be a partially normalised program, let $Q$ be a query and let $q$ be $\operatorname{rel}(Q)$.

1. For each $p \simeq q$ construct $\mathcal{A}_{p}$.

2. Adorn $P$ with respect to $q$ and $\bigcup_{p \simeq q} \mathcal{A}_{p}$.

3. Let $A=\left\{a \mid a \in \mathcal{A}_{q}\right.$, for all $p$ such that $q^{a} \sqsupseteq p: p$ is not recursive in $\left.P^{a}\right\}$.

Let $c_{1}=\bigvee_{a \in A}$. Let $c_{2}=\bigvee_{a \in \mathcal{A}_{q}, a \notin A}$.

4. Remove "irrelevant clauses"

Let $A_{1}, \ldots, A_{n} \in \mathcal{A}_{q}$ be the only consistent with $c_{2}$ adornments of $q$. For every rule $r$ in $P^{a}$

If for all $i, q^{A_{i}} \nsupseteq \operatorname{rel}(\operatorname{Head}(r))$ remove $r$ from $P^{a}$

5. Define a symbolic counterparts of norms,

level mappings and interargument relations.

6. Construct constraints on the symbolic variables. Obtain $S$.

7. Solve $S$.

(a) Solution of $S$ doesn't produce extra constraints on variables. Report termination for $c_{1} \vee c_{2}$.

(b) Solution of $S$ produces extra constraints involving new integer variables. Conjunct these constraints to termination condition $c_{2}$. Go back to step 2.

(c) Otherwise report termination for $c_{1}$.

Fig. 1. Termination Inference Algorithm

that is $c_{\$ 1>\$ 2} Y>0$. Since $c_{\$ 1>\$ 2} \geq 0, Y>0$ and $c_{\$ 1>\$ 2}>0$ should hold. We update $c_{2}$ to be $(\$ 1>\$ 2) \wedge(\$ 2>0)$. Now we restart the whole process with respect to $Y>0$. The following adorned program is obtained: $q^{\$ 1>\$ 2, \$ 2>0}(X, Y) \leftarrow X>Y, Z$ is $X-Y, q^{\$ 1>\$ 2, \$ 2>0}(Z, Y)$. $q^{\$ 1>\$ 2, \$ 2 \leq 0}(X, Y) \leftarrow X>Y, Z$ is $X-Y, q^{\$ 1>\$ 2, \$ 2 \leq 0}(Z, Y)$. $q^{\$ 1>\$ 2, \$ 2 \leq 0}(X, Y) \leftarrow X>Y, Z$ is $X-Y, q^{\$ 1 \leq \$ 2, \$ 2 \leq 0}(Z, Y)$.

The second and the third clauses are removed, since they are "irrelevant" with respect to $\$ 2>0$. The level mapping is redefined as

$$
\left|q^{\$ 1>\$ 2}(X, Y)\right|=c_{\$ 1>\$ 2} *\left\{\begin{array}{ll}
X-Y & \text { if } X>Y \\
0 & \text { otherwise }
\end{array}+c_{\$ 2>0} *\left\{\begin{array}{l}
Y \text { if } Y>0 \\
0 \text { otherwise }
\end{array}\right.\right.
$$

Acceptability decreases imply $c_{\$ 1>\$ 2}(X-Y)+c_{\$ 2>0} Y>c_{\$ 1>\$ 2}((X-$ $Y)-Y)+c_{\$ 2>0} Y$, i.e., $0>-c_{\$ 1>\$ 2} Y$. The inequality holds, since $Y>$ 0 and $c_{\$ 1>\$ 2}>0$ are assumed to hold. This solution does not impose additional constraints on integer variables. Thus, the analysis terminates reporting $\$ 1 \leq \$ 2 \vee(\$ 1>\$ 2 \wedge \$ 2>0)$ as a termination condition.

In order to prove correctness of this algorithm we have to prove its termination and partial correctness. Termination follows from termination 
of its steps and from the finiteness of the number of integer variables, restricting a number of backwards steps from 7(b) to 2. Partial correctness follows from the correctness of transformations and [7].

\section{$5 \quad$ Further extensions}

In this section we discuss possible extensions of the algorithm presented above. First of all, we re-consider inference of adornments, then we discuss integrating termination analysis of numerical and symbolic computations.

\subsection{Once more about the inference of adornments}

The set of adornments $\mathcal{A}_{p}$, inferred in Subsection 4.1 may sometimes be too weak for inferring precise termination conditions.

Example 15. $p(X, Y) \leftarrow X<0, Y 1$ is $Y+1, X 1$ is $X-1, p(Y 1, X 1)$. The maximal prefix of the rule above is $X<0$, thus, $\mathcal{C}_{p}=\{\$ 1<0\}$ and $\mathcal{A}_{p}=\{\$ 1<0, \$ 1 \geq 0\}$. The only termination condition found is $\$ 1 \geq 0$, while the precise termination condition is $\$ 1 \geq 0 \vee(\$ 1<0 \wedge \$ 2 \geq-1)$.

The problem occured due to the fact that $\mathcal{A}_{p}$ restricts only some subset of integer argument positions, while for the termination proof information on integer arguments outside of this subset may be needed.

Definition 15. Let $P$ be a program, let $p$ be a predicate in $P$, let $C_{q}$ be a set of symbolic conditions over the integer argument positions of $q$, and $C=\cup_{q \in P} C_{q}$. A symbolic condition c over the integer argument positions of $p$ is called an extension of $C$ if there exists $r \in P$, defining $p$, such that some integer argument position denominator appearing in $c$ does not appear in $C_{p}$, and $c$ is implied by some $c_{q} \in C_{q}$ for the recursive subgoals and some interargument relations for the non-recursive ones.

Let $C$ be a set of symbolic conditions over the integer argument positions of $p$ and let $\varphi(C)$ be $C \cup\{c \mid c$ is an extension of $C\}$. Define the set of adornments for $p$ as $\left\{c_{1}^{\prime} \wedge \ldots \wedge c_{n}^{\prime} \mid c_{i}^{\prime} \in \varphi^{*}\left(\mathcal{C}_{p}\right)\right.$ or $\left.\neg c_{i}^{\prime} \in \varphi^{*}\left(\mathcal{C}_{p}\right)\right\}$, where $\varphi^{*}$ is a fixpoint of powers of $\varphi$ and $\mathcal{C}_{p}$ is defined as in Subsection 4.1.

Example 16. Example 15, continued. The only extension of $\mathcal{C}_{p}$ is $\$ 1<0 \wedge$ $\$ 2<-1$, i.e., $\varphi\left(\mathcal{C}_{p}\right)=\{\$ 1<0, \$ 1<0 \wedge \$ 2<-1\}$. Thus, $\varphi^{*}\left(\mathcal{C}_{p}\right)=\varphi\left(\mathcal{C}_{p}\right)$ and $\mathcal{A}_{p}=\{\$ 1<0 \wedge \$ 2<-1,(\$ 1<0 \wedge \$ 2 \geq-1) \vee \$ 1 \geq 0\}$.

An alternative approach to propagating such an information was suggested in [8]. It allows one to propagate the existing adornments but not to infer the new ones and thus, is less precise than our approach. 


\subsection{Integrating numerical and symbolic computation}

In the real-world programs numerical computations are sometimes interleaved with symbolic ones, as illustrated by the following example [11].

Example 1\%.

$$
\begin{array}{ll}
\operatorname{collect}(X,[X \mid L], L) \leftarrow \operatorname{atomic}(X) . & \operatorname{process}(-, A, A, L, L) . \\
\operatorname{collect}(T, L 0, L) \leftarrow & \operatorname{process}(T, I, A, L 0, L 2) \leftarrow \\
\operatorname{compound}(T), \text { functor }\left(T,,_{-}, A\right), & I<A, I 1 \text { is } I+1, \arg (I 1, T, A r g), \\
\operatorname{process}(T, 0, A, L 0, L) . & \operatorname{collect}(\operatorname{Arg}, L 0, L 1), \\
& \operatorname{process}(T, I 1, A, L 1, L 2) .
\end{array}
$$

To prove termination of $\{$ collect (tree, variable, [])\} three decreases should be shown: between a call to collect and a call to process between a call to process and a call to collect and between two calls to process. The first two can be shown only by a symbolic level mapping, the third one - only by the numerical approach.

Thus, our goal is to combine the existing symbolic approaches with the numerical one presented so far. One of the possible ways to do so is to combine two level mappings, $|\cdot|_{1}$ and $|\cdot|_{2}$ by mapping each atom $A \in B_{P}^{E}$ to a pair of natural numbers $\left(|A|_{1},|A|_{2}\right)$ and prove termination by establishing decreases on orderings of such pairs [14].

Example 18. Example 17, continued. Define $\varphi: B_{P}^{E} \rightarrow\left(\mathcal{N} \cup \mathcal{N}^{2}\right)$ as: $\varphi(\operatorname{collect}(t, l 0, l))=\|t\|, \varphi(\operatorname{process}(t, i, a, l 0, l))=(\|t\|, a-i)$ where $\|\cdot\|$ is a term-size norm. The decreases are satisfied with respect to $>$, such that $A_{1}>A_{2}$ if and only if $\varphi\left(A_{1}\right) \succ \varphi\left(A_{2}\right)$, where $\succ$ is defined as: $n \succ m$, if $n>_{\mathcal{N}} m, n \succ(n, m)$, if true, $\left(n, m_{1}\right) \succ\left(n, m_{2}\right)$, if $m_{1}>_{\mathcal{N}} m_{2}$ and $\left(n_{1}, m\right) \succ n_{2}$, if $n_{1}>_{\mathcal{N}} n_{2}$ and $>_{\mathcal{N}}$ is the usual order on the naturals.

This integrated approach allows one to analyse correctly examples such as ground, unify, numbervars [15] and Example 6.12 in [8].

\section{Conclusion}

Termination of numerical computations was studied by a number of authors [1,2,8]. Apt et al. [2] provided a declarative semantics, so called $\Theta$-semantics, for Prolog programs with first-order built-in predicates, including arithmetic operations. In this framework the property of strong termination, i.e., finiteness of all LD-trees for all possible goals, was completely characterised based on appropriately tuned notion of acceptability. This approach provides important theoretical results, but seems to 
be difficult to integrate in automatic tools. In [1] it is claimed that an unchanged acceptability condition can be applied to programs in pure Prolog with arithmetic by defining the level mappings on ground atoms with the arithmetic relation to be zero. This approach ignores the actual computation, and thus, its applicability is restricted to programs using some arithmetic but not really relaying on them, such as quicksort. Moreover, as Example 14 illustrates, there are many programs that terminate only for some queries. Alternatively, Dershowitz et al. [8] extended the query-mapping pairs formalism of [9] to deal with numerical computations. However, this approach inherited the disadvantages of [9], such as high computational price.

More research has been done on termination analysis for constraint logic programs [4, 10,12]. Since numerical computations in Prolog should be written in a way that allows a system to verify their satisfiability we can see numerical computations of Prolog as an ideal constraint system. Thus, all the results obtained for ideal constraints systems can be applied. Unfortunately, the research was either oriented towards theoretical characterisations [12] or restricted to domains isomorphic to $\mathcal{N}$ [10].

In a contrast to the approach of [8] that was restricted to verifying termination, we presented a methodology for inferring termination conditions. It is not clear whether and how [8] can be extended to infer such conditions. A main contribution of this work to the theoretical understanding of termination of numerical computations is in situating them in the well-known framework of acceptability and allowing integration with the existing approaches to termination of symbolic computations. The methodology presented can be integrated in automatic termination analysers, such as [7].

The kernel technique is powerful enough to analyse correctly examples such as gcd and mod [8] and all examples appearing in Chapter 8 of [15], which is dedicated to arithmetic. These examples include the examples appearing in [1]. Moreover, our approach gains its power from the underlying framework of [7] and thus, allows one to prove termination of some examples that cannot be analysed correctly by [8], similar to confused delete [7]. The extended technique, presented in Section 5, allows one to analyse correctly examples such as Ackermann's function, ground, unify, numbervars [15] and Example 6.12 in [8].

As future work we consider a complete implementation of the algorithm. Due to the use of the constraint solving techniques we expect it both to be powerful and highly efficient. 


\section{References}

1. K. R. Apt. From Logic Programming to Prolog. Prentice-Hall Int. Series in Computer Science. Prentice Hall, 1997.

2. K. R. Apt, E. Marchiori, and C. Palamidessi. A declarative approach for firstorder built-in's in prolog. Applicable Algebra in Engineering, Communication and Computation, 5(3/4):159-191, 1994.

3. A. Bossi and N. Cocco. Preserving universal temination through unfold/fold. In G. Levi and M. Rodríguez-Artalejo, editors, Algebraic and Logic Programming, pages 269-286. Springer Verlag, 1994. LNCS 850.

4. L. Colussi, E. Marchiori, and M. Marchiori. On termination of constraint logic programs. In U. Montanari and F. Rossi, editors, Principles and Practice of Constraint Programming - CP'95,, pages 431-448. Springer Verlag, 1995. LNCS 976.

5. D. De Schreye, K. Verschaetse, and M. Bruynooghe. A framework for analyzing the termination of definite logic programs with respect to call patterns. In I. Staff, editor, Proc. of the Int. Conf. on Fifth Generation Computer Systems., pages 481488. IOS Press, 1992.

6. S. Decorte and D. De Schreye. Termination analysis: some practical properties of the norm and level mapping space. In J. Jaffar, editor, Proc. of the 1998 Joint Int. Conf. and Symp. on Logic Programming, pages 235-249. MIT Press, June 1998.

7. S. Decorte, D. De Schreye, and H. Vandecasteele. Constraint-based termination analysis of logic programs. ACM Transactions on Programming Languages and Systems (TOPLAS), 21(6):1137-1195, November 1999.

8. N. Dershowitz, N. Lindenstrauss, Y. Sagiv, and A. Serebrenik. A general framework for automatic termination analysis of logic programs. Applicable Algebra in Engineering, Communication and Computing, 12(1-2):117-156, 2001.

9. N. Lindenstrauss and Y. Sagiv. Automatic termination analysis of logic programs. In L. Naish, editor, Proc. of the Fourteenth Int. Conf. on Logic Programming, pages 63-77. MIT Press, July 1997.

10. F. Mesnard. Inferring left-terminating classes of queries for constraint logic programs. In M. Maher, editor, Proc. JICSLP'96, pages 7-21. The MIT Press, 1996.

11. C. Pollard and I. A. Sag. Head-driven Phrase Structure Grammar. The University of Chicago Press, 1994.

12. S. Ruggieri. Verification and validation of logic programs. $\mathrm{PhD}$ thesis, Universitá di Pisa, 1999

13. A. Serebrenik and D. De Schreye. Inference of termination conditions for numerical loops. Technical Report CW 308, Departement Computerwetenschappen, K.U.Leuven, Leuven, Belgium, 2001.

14. A. Serebrenik and D. De Schreye. Non-transformational termination analysis of logic programs, based on general term-orderings. In K.-K. Lau, editor, Logic Based Program Synthesis and Transformation 10th International Workshop, Selected Papers, volume 2042 of Lecture Notes in Computer Science, pages 69-85. Springer Verlag, 2001.

15. L. Sterling and E. Shapiro. The Art of Prolog. The MIT Press, 1994. 